\title{
Potential role of recombinant adeno-associated virus human thioredoxin-PR39 in cell and vascular protection against hypoxia
}

\author{
XI-YUN RUAN ${ }^{1}$, YING-CHUN LIANG ${ }^{2}$, BIN DU $^{3}$, YOU-TING LIN ${ }^{1}$, YU-DONG GUO $^{4}$, \\ JING ZHAO ${ }^{5}$, SHAN LI ${ }^{1}$, JI-FENG LI ${ }^{1}$, QIN-JIAN SUN ${ }^{1}$ and YI-FENG DU ${ }^{1}$ \\ ${ }^{1}$ Department of Neurology, Shandong Provincial Hospital Affiliated to Shandong University, Jinan, Shandong 250021; \\ ${ }^{2}$ Department of Neurology, The Central Hospital of Tai'an, Tai'an, Shandong 271000; ${ }^{3}$ Department of Neurosurgery, \\ Jinan Central Hospital Affiliated to Shandong University; ${ }^{4}$ Department of Neurosurgery, The Fifth Hospital of Jinan; \\ ${ }^{5}$ Department of Neurology, Jinan Central Hospital Affiliated to Shandong University, Jinan, Shandong 250021, P.R. China
}

Received July 7, 2014; Accepted January 16, 2015

DOI: 10.3892/etm.2015.2301

\begin{abstract}
The aim of the present study was to successfully construct a recombinant adeno-associated virus (rAAV) vector containing the human thioredoxin (hTRX)-PR39 chimeric gene (rAAV/hTRX-PR39), and verify that the vector was able to maintain a sustained, stable and efficient expression to achieve protein production in the cell. In the present study, a chicken embryo model was utilized to analyze the therapeutical effect of rAAV/hTRX-PR39 in cerebral ischemia diseases. ECV304 cells were transfected with rAAV/hTRX-PR39 and incubated under conditions of 20,5 and $1 \% \mathrm{O}_{2}$. Subsequently, the expression levels of vascular endothelial growth factor (VEGF), vascular endothelial growth factor receptor (VEGFR)-1, VEGFR-2, fibroblast growth factor receptor (FGFR)-1 and syndecan-4 were detected by reverse transcription-quantitative polymerase chain reaction. Under hypoxic conditions, the mRNA expression levels of VEGF, VEGFR-1, VEGFR-2, FGFR-1 and syndecan-4 were found to increase in the PR39-transfected group when compared with the control group, while no statistically significant difference was observed between the PR39-transfected group and the control group under conditions of $20 \% \mathrm{O}_{2}$. In addition, hTRX-PR39 was shown to increase the density of the vasculature and the survival rate of the chick embryos. Under hypoxic conditions, it was hypothesized that rAAV/hTRX-PR39 was capable of promoting angiogenesis, which may subsequently protect the cells from impairment by hypoxia. In conclusion, rAAV/hTRX-PR39 was demonstrated to promote vasculariza-
\end{abstract}

Correspondence to: Professor Qin-Jian Sun, Department of Neurology, Shandong Provincial Hospital Affiliated to Shandong University, 324 Jingwu Weiqi Road, Jinan, Shandong 250021, P.R. China

E-mail: qinjiansundoc@163.com

Key words: hypoxia, ischemia, recombinant adeno-associated virus/human thioredoxin-PR39, angiogenesis, gene therapy tion and cell survival in hypoxia; thus, rAAV/hTRX-PR39 may have potential for use in therapy targeting cerebral ischemia.

\section{Introduction}

Cerebral infarction is a severe disease of the central nervous system that has a high incidence rate. Thrombolytic therapy is considered to be the only safe and effective method to recover the blood supply; however, an effective method of providing neuronal protection against ischemia and ischemia-reperfusion injury has not yet been identified. In addition, a method for inducing angiogenesis, which may aid the establishment of collateral circulation and secondary prevention, has not yet been developed (1).

Gene therapy has been considered to be prospective in the treatment of infarction and neuron protection $(2,3)$. A number of studies assessing monogenic function have been conducted, where the protective roles of neurotrophic factors, antiapoptosis genes and angiogenic growth factors have been investigated (4). However, the mechanisms underlying cerebral ischemic diseases are complicated and involve a variety of courses; thus, it was proposed that regulating two genes that exhibit positive feedback on each other may aid further understanding (5). Human thioredoxin (hTRX) is a type of stress-induced protein that protects neurons against oxidative stress $(6,7)$. hTRX has been demonstrated to possess the ability to scavenge free radicals, and also ease the inflammatory response by regulating nuclear factors or mitogen-activated protein kinases. In addition, since hTRX is a type of natural human protein with low immunogenicity, hTRX can be used as a frame protein to construct a gene fusion system, in which the solubility and activity of the expression product can be increased significantly $(8,9)$.

Antibacterial peptide (PR39), a proline- and arginine-rich peptide consisting of 39 amino acids, is considered to be the switch for angiogenesis (10). PR39 is able to inhibit the degradation of hypoxia inducible factor (HIF)- $1 \alpha$, which subsequently elevates the expression of vascular endothelial growth factor (VEGF), kinase insert domain containing-receptor, fms-like tyrosine kinase and fibroblast growth factor receptor (FGFR)-1 to promote vascularization $(11,12)$. This mechanism 
of action is similar to the mechanism of vascularization that is observed under conditions of hypoxia. Sun et al indicated that adeno-associated virus (AAV)-PR39 may serve as a novel therapeutic agent for the treatment of myocardial infarction (13). As a short peptide, PR39 is unstable; thus, hTRX may be used to provide a frame structure for PR39. Following the insertion of the hTRX frame structure, the aptamer (PR39) is more stable compared with the free peptide. Furthermore, the cell-penetrating ability of hTRX $(14,15)$ may enable PR39 to pass through the blood-brain barrier, which is conducive to enabling the full function of PR39.

In the present study, it was hypothesized that the recombinant gene, hTRX-PR39, may exhibit multiple functions in the protection of neurons and the vasculature. Thus, the aim of the present study was to investigate the therapeutic roles of hTRX-PR39 in hypoxia.

\section{Materials and methods}

Recombinant virus construction. The pGEM-T-hTRX-PR39 cloning vector containing hTRX-PR39 full-length gene sequence was constructed as previously described (16). First, the forward and reverse primers of PR39 were designed and synthesized. Using PCR, the fragment encoding PR39 was produced, including EcoR721 and BamHI restriction enzyme sites, and the new hTRX cDNA including EcoR721 and $E c o$ RI restriction enzyme sites was generated. Next, the synthesized fragments were cloned into a pGEM-T vector. The positive clone was identified using restriction enzymes, and the cloned amplified fragments were sequenced by the dideoxy-mediated chain-termination method. The cloned hTRX and PR39 cDNA were compared with the GenBank sequence (http://www.ncbi.nlm.nih.gov/genbank/) using DNASIS software (MiraiBio Group, San Francisco, CA, USA). Subsequently, pGEM-T-hTRX and pGEM-T-PR39 were digested by BamHI and EcoRI, the PR39 BamHI, EcoRI was cloned into the recombinant vector pGEM-T-hTRX BamHI, EcoRI. Thus, the recombinant vector pGEM-T-hTRX-PR39 was produced. The pSSCMV viral vector, adenovirus plasmid pAAV/Ad, Escherichia coli TOP10, ECV304 and HEK293 cell lines were provided by Xi'an Huaguang Biological Engineering Co., Ltd. (Xi'an, China) (17).

Transfection. The recombinant virus was seeded into the culture medium of ECV304 cells (Xi'an Huaguang Biological Engineering Co., Ltd.) and incubated for 24 h. For the control, adenoviruses were seeded into the culture medium of ECV304 cells instead of the recombinant virus. The control and transfection groups were subsequently divided into three subgroups that were separately incubated in a hypoxic $\left(1\right.$ and $\left.5 \% \mathrm{O}_{2}\right)$ or normoxic environment $\left(20 \% \mathrm{O}_{2}\right)$ for $72 \mathrm{~h}$.

Reverse transcription-quantitative polymerase chain reaction (PCR). Total RNA was extracted using TRIzol reagent (Invitrogen Life Technologies, Carlsbad, CA, USA) and reverse transcribed to cDNA using a Moloney Murine Leukemia Virus (M-MLV) reverse transcriptase PCR kit (Promega Corporation, Madison, WI, USA). Briefly, $3 \mu$ l RNA was reverse transcribed to cDNA at $37^{\circ} \mathrm{C}$ for $1 \mathrm{~h}$ in a $20-\mu 1$ reaction system that contained $1 \mu 1 \mathrm{M}-\mathrm{MLV}$ reverse transcriptase,
$4 \mu \mathrm{l}$ 5X M-MLV buffer, $0.5 \mu \mathrm{l}$ RNase inhibitor, $1 \mu \mathrm{l}$ oligo-dT and $1 \mu \mathrm{ldNTP}$ (Promega Corporation, Madison, WI, USA). For quantitative PCR, the PCR amplification mixture $(20 \mu \mathrm{l})$ consisted of $2 \mu \mathrm{l}$ cDNA mixture, $10 \mu \mathrm{l} \mathrm{SYBR}$ Green (Takara Biotechnology Co., Ltd., Dalian, China), $2 \mu \mathrm{l}$ primers and $6 \mu \mathrm{l}$ deionized water. $\beta$-actin was used as a control. The amplification conditions were as follows: Initial denaturation at $95^{\circ} \mathrm{C}$ for $2 \mathrm{~min}$, followed by 40 cycles of $95^{\circ} \mathrm{C}$ for $10 \mathrm{sec}, 58^{\circ} \mathrm{C}$ for $30 \mathrm{sec}$ and $72^{\circ} \mathrm{C}$ for $30 \mathrm{sec}$. Nested PCR was performed using a $2-\mu 1$ sample of the PCR product as a template under the aforementioned PCR conditions. Bio-Rad IQ5.0 Optical System software (Bio-Rad Laboratories, Hercules, CA, USA) was used for the detection of the quantitative PCR products that were specific for VEGF, vascular endothelial growth factor receptor (VEGFR)-1, VEGFR-2, FGFR-1, syndecan-4, PR39 and $\beta$-actin. The primer sequences used for PCR are shown in Table I.

Effects of AAV-hTRX-PR39 transfection on the hypoxic chick embryo. Effects of AAV-hTRX-PR39 on the hypoxic chick embryo were analyzed using a chick embryo (Xi'an Huaguang Biological Engineering Co., Ltd.) chorioallantoic membrane (CAM). In total, 120 fertilized chicken eggs (age, seven days; weight, 50-55 g) were incubated under $65-70 \%$ relative air humidity at $37^{\circ} \mathrm{C}$. On day four of incubation, $100 \mu \mathrm{l}$ AAV-hTRX-PR39 or adenovirus (control) was gently pipetted onto the CAM surface using a transfer pipette. The eggs were subsequently placed in an incubator for three days. Next, the CAMs were incubated for $8 \mathrm{~h}$ in a hypoxic $\left(1\right.$ and $\left.5 \% \mathrm{O}_{2}\right)$ or normoxic $\left(20 \% \mathrm{O}_{2}\right)$ environment, after which they were incubated for 11 days in a normoxic environment. The CAMs were subsequently photographed using an Olympus DP73 digital camera (Olympus Corporation, Tokyo, Japan), as presented in Fig. 1. The vessel density of the CAMs was analyzed using Image-Pro Plus software (Media Cybernetics, Inc., Rockville, MD, USA). For each study group, 10-15 domains were selected for vessel quantification, and the mean values of the vessel density were calculated. All animal studies were approved by the Shandong University Institutional Animal Care and Use Committee.

Statistical analysis. Data are expressed as the mean \pm standard deviation, and were analyzed using analysis of variance and the homogeneity of variance test, according to a completely randomized design. Comparisons between groups were performed using the two-sample t-test. SPSS software, version 13.0 (SPSS, Inc., Chicago, IL, USA) was used for statistical analysis, and $\mathrm{P}<0.05$ was considered to indicate a statistically significant difference.

\section{Results}

Expression levels of VEGF, VEGFR-1, VEGFR-2, FGFR-1, syndecan-4 and PR39. Under normoxic conditions of $20 \% \mathrm{O}_{2}$, the mRNA expression levels of PR39, VEGF, VEGFR-1, VEGFR-2, FGFR-1 and syndecan-4 exhibited no statistically significant difference when comparing the hTRX-PR39-transfected ECV304 cells and the control group $(\mathrm{P}>0.05)$. However, under hypoxic conditions of $1 \% \mathrm{O}_{2}$, the quantitative $\mathrm{PCR}$ results demonstrated increased mRNA 
Table I. Primers used for reverse transcription-quantitative polymerase chain reaction.

\begin{tabular}{lllr}
\hline Genes & \multicolumn{1}{c}{ Forward primer (5'-3') } & Reverse primer (5'-3') & Product (bp) \\
\hline VEGF & TCTACCTCCACCATGCCAAGT & GCTGCGCTGATAGACATCCA & 104 \\
VEGFR-1 & TCCCTTATGATGCCAGCAAGT & CCAAAAGCCCCTCTTCCAA & 79 \\
VEGFR-2 & CTTCGAAGCATCAGCATAAGAAACT & TGGTCATCAGCCCACTGGAT & 156 \\
FGFR-1 & ACTCTGTGGTGCCTTCTGAC & CATTTCCTTGTCGGTGGTAT & 317 \\
Syndecan-4 & CTGCTGCTGTTCTTCGTAGG & CTTTGAGCTGTCTGGCTCTG & 153 \\
PR39 & CTCTACCGCCTCCTGGAGCT & GGCCCTTCATAATATCCCCCA & 117 \\
\hline
\end{tabular}

VEGF, vascular endothelial growth factor; VEGFR, vascular endothelial growth factor receptor, FGFR, fibroblast growth factor receptor.

Table II. Expression levels of the angiogenic growth factors in the control and PR39-transfected groups.

\begin{tabular}{|c|c|c|c|c|}
\hline \multirow[b]{2}{*}{ Gene expression } & \multicolumn{2}{|c|}{$20 \% \mathrm{O}_{2}$} & \multicolumn{2}{|c|}{$1 \% \mathrm{O}_{2}$} \\
\hline & $\begin{array}{l}\text { Control } 1 \\
\text { group }\end{array}$ & PR39 & $\begin{array}{c}\text { Control } 2 \\
\text { group }\end{array}$ & PR39 \\
\hline VEGF & $0.3 \pm 0.06$ & $0.33 \pm 0.08$ & $0.47 \pm 0.22$ & $0.75 \pm 0.25^{\mathrm{a}}$ \\
\hline VEGFR-1 & $0.14 \pm 0.07$ & $0.15 \pm 0.10$ & $0.31 \pm 0.09$ & $0.49 \pm 0.21^{\mathrm{a}}$ \\
\hline VEGFR-2 & $0.16 \pm 0.05$ & $0.15 \pm 0.11$ & $0.35 \pm 0.10$ & $0.54 \pm 0.12^{\mathrm{a}}$ \\
\hline FGFR-1 & $0.23 \pm 0.06$ & $0.28 \pm 0.16$ & $0.42 \pm 0.14$ & $0.67 \pm 0.20^{\mathrm{a}}$ \\
\hline Syndecan-4 & $0.16 \pm 0.05$ & $0.15 \pm 0.04$ & $0.29 \pm 0.06$ & $0.39 \pm 0.11^{\mathrm{a}}$ \\
\hline PR39 & $0.00 \pm 0.00$ & $0.10 \pm 0.12$ & $0.00 \pm 0.00$ & $1.43 \pm 0.25^{\mathrm{a}}$ \\
\hline
\end{tabular}

${ }^{\mathrm{a} P}<0.05$, vs. control 2 group. VEGF, vascular endothelial growth factor; VEGFR, vascular endothelial growth factor receptor; FGFR, fibroblast growth factor receptor.

Table III. Effects of AAV-hTRX-PR39 transfection on the hypoxic chick embryo.

\begin{tabular}{|c|c|c|c|}
\hline Group & Survival rate (n) & Wet weight (g) & Density of vessels (\%) \\
\hline \multicolumn{4}{|l|}{$1 \% \mathrm{O}_{2}$} \\
\hline Control 1 & $0 / 20^{c}$ & $8.5 \pm 3.56^{\mathrm{c}}$ & $2.21 \pm 0.4^{\mathrm{c}}$ \\
\hline PR39 & $16 / 20^{\mathrm{a}}$ & $32.5 \pm 4.5^{\mathrm{a}}$ & $10.6 \pm 0.6^{\mathrm{a}}$ \\
\hline \multicolumn{4}{|l|}{$5 \% \mathrm{O}_{2}$} \\
\hline Control 2 & $3 / 20^{c}$ & $20.4 \pm 8.56^{\mathrm{c}}$ & $5.65 \pm 0.6^{\mathrm{c}}$ \\
\hline PR39 & $17 / 20^{\mathrm{b}}$ & $34.31 \pm 6.51^{\mathrm{b}}$ & $11.9 \pm 0.5^{\mathrm{b}}$ \\
\hline \multicolumn{4}{|l|}{$20 \% \mathrm{O}_{2}$} \\
\hline Control 3 & $20 / 20$ & $38.8 \pm 4.27$ & $12.5 \pm 0.5$ \\
\hline PR39 & $20 / 20$ & $34.2 \pm 5.44$ & $10.1 \pm 0.5$ \\
\hline
\end{tabular}

${ }^{\mathrm{a}} \mathrm{P}<0.05$, vs. control 1 group; ${ }^{\mathrm{b}} \mathrm{P}<0.05$, vs. control 2 group; and ${ }^{\mathrm{c}} \mathrm{P}<0.05$, vs. control 3 group. $\mathrm{AAV}$, adeno-associated virus; hTRX, human thioredoxin.

expression levels of VEGF, VEGFR-1, VEGFR-2, FGFR-1 and syndecan-4, as well as sharply increased expression levels of PR39, in the hTRX-PR39-transfected group $(\mathrm{P}<0.05)$, as compared with the control group. The results are shown in Table II. The $5 \% \mathrm{O}_{2}$ condition was not examined as the $20 \%$ and $1 \% \mathrm{O}_{2}$ conditions successfully demonstrated that transfection with $\mathrm{rAAV} / \mathrm{hTRX}$-PR39 increased the expression levels of
VEGF, VEGFR-1, VEGFR-2, FGFR-1 and syndecan-4 under hypoxic condition $\left(1 \% \mathrm{O}_{2}\right)$.

Effects of AAV-hTRX-PR39 transfection on the hypoxic chick embryo. Under $20 \% \mathrm{O}_{2}$ conditions, the survival rate of the chick embryos was $100 \%$ in the transfected and non-transfected groups $(\mathrm{P}>0.05)$. By contrast, under hypoxic conditions (5 or 



Figure 1. Effect of adeno-associated virus-human thioredoxin-PR39 transfection on the hypoxic chick embryo in various $\mathrm{O}_{2}$ environments. (A) $1 \% \mathrm{O}_{2}$, (B) $1 \%$ $\mathrm{O}_{2} \mathrm{PR} 39$, (C) $5 \% \mathrm{O}_{2}$, (D) $5 \% \mathrm{O}_{2} \mathrm{PR} 39$, (E) $20 \% \mathrm{O}_{2}$ and (F) $20 \% \mathrm{O}_{2} \mathrm{PR} 39$.

$1 \% \mathrm{O}_{2}$ ), the survival rate of the chick embryos was shown to increase in the transfected group when compared with the non-transfected group $\left(5 \% \mathrm{O}_{2}, 17 / 20\right.$ vs. $3 / 20 ; 1 \% \mathrm{O}_{2}, 16 / 20$ vs. $0 / 20 ; \mathrm{P}<0.05)$.

As shown in Table III, the wet weight of the chick embryos under normoxic conditions did not significantly differ between the transfected or non-transfected groups $(\mathrm{P}>0.05)$. However, the wet weight of the chick embryos under hypoxic conditions (5 or $1 \% \mathrm{O}_{2}$ ) increased significantly in the transfected group when compared with the non-transfected group $(\mathrm{P}<0.05)$.

Under a normoxic environment, no statistically significant difference was observed with regard to the density of the vessels between the transfected and non-transfected groups $(\mathrm{P}>0.05)$. However, the density of the vessels in the chick embryos subjected to mild hypoxia $\left(5 \% \mathrm{O}_{2}\right)$ and severe hypoxia $\left(1 \% \mathrm{O}_{2}\right)$ decreased significantly in the non-transfected group when compared with the $20 \% \mathrm{O}_{2}$ control group. In addition, the density of the vasculature increased significantly in the transfected groups when compared with the respective non-transfected groups $(\mathrm{P}<0.05)$. The results are presented in Table III.

\section{Discussion}

hTRX is a micromolecular protein that functions as an oxidant and a reductant. The hTRX gene is $13 \mathrm{~kb}$ in length and encodes 104 amino acids (18). hTRX is known to play a number of important roles, including regulating the redox reaction, scavenging free radicals and exerting antiapoptosis effects (19-21). Previously, hTRX protein homology was demonstrated to consist of a protein cross-frame feature that provides available sites for the binding of an active aptamer $(9,22)$. Following the insertion of the aptamer into the cross-frame structure, the aptamer becomes more stable compared with the free peptide and is more prone to transfer into cells. PR 39, a short peptide that is extremely unstable, is prone to inactivating conformational changes. Thus, therapeutic use of PR39 requires expression as a recombinant protein. The hTRX protein can provide a framework for the expression of PR39 as a therapeutic aptamer. As hTRX is a natural human protein with low immunogenicity, hTRX can serve as a cross-frame protein for the construction of a gene fusion expression system, which may significantly 
increase the activity of the expression products and activated soluble proteins. Previous studies have demonstrated that hTRX-PR39 can reduce the number of apoptotic ECV304 cells under hypoxic conditions $(23,24)$. Thus, the hTRX-PR39 chimeric protein provides structural compatibility to ensure the directed bioavailability of PR39 at the target site, in addition to the added stability of the protein. Furthermore, in the present study, the mRNA expression levels of PR39 and the various growth factors were only activated under conditions of hypoxia, but not under conditions of $20 \% \mathrm{O}_{2}$, indicating that the application of hTRX-PR39 is controllable.

In the present study, the mRNA expression levels of VEGF, VEGFR-2, FGFR-1 and syndecan-4 were shown to increase in the PR39-transfected groups when compared with the respective control groups, indicating that PR39 may activate these growth factors and receptors. To assess the effect on vascularization, the density of the allantoic sac vasculature were calculated. The results demonstrated that the density of the vasculature was markedly increased in the transfected groups. Accordingly, the survival rates of the chick embryos were also improved in the transfected groups when compared with the respective control groups. Therefore, the results indicated that hTRX-PR39 was able to induce tolerance to hypoxia.

PR39 has been previously demonstrated to prevent the degradation of HIF-1 $\alpha$, which results in the upregulation of HIF-1 $\alpha$-dependent genes, including VEGF and VEGFR-1 $(24,25)$. In addition, PR39 is known to upregulate the expression of the FGFRs, FGFR-1 and syndecan-4, which subsequently activate FGF signaling $(24,26)$. The improvement in blood supply may further increased the level of PR39 (27,28). In the present study, increases in the expression levels of angiogenic growth factors and vascularization were confirmed in the PR39-transfected groups; thus, it was hypothesized that these factors may be the main mechanisms underlying the protective role of PR39 in hypoxia. In addition, PR39 has been hypothesized to play a role in the protection of IAP-2 (an inhibitor of apoptosis) and the decreased activity of caspase-3, leading to the suppression of apoptosis (29). According to these results, PR39 may stimulate an angiogenic response, which may be used as a therapeutic intervention in ischemic areas. In future studies, hTRX-PR39 should be transfected into ischemic brain tissues to observe the protective role in ischemic stroke.

According to previous studies (30-33) by other groups, and our previous study (34), a recombinant AVV (rAAV) vector, containing the hTRX-PR39 chimeric gene, was constructed to achieve sustained, stable and efficient protein production. Vectors containing hTRX-PR39 were transfected into chick embryonic tissues that had been subjected to hypoxia, and the expression of PR39 was shown to be activated, as well as that of downstream factors. Expression activation was only observed under hypoxic conditions, indicating that the expression is well-controlled. Thus, rAAV/hTRX-PR39 was demonstrated to promote vascularization and cell survival in hypoxia, and may have potential in the therapy of cerebral ischemia.

In conclusion, the hTRX-PR39 chimeric protein provides structural compatibility to ensure the directed bioavailability of PR39 at the target site, in addition to added stability of the protein. Furthermore, transfection with rAAV/hTRX-PR39 was shown to increase the expression levels of VEGF,
VEGFR-1, VEGFR-2, FGFR-1 and syndecan-4, and promote angiogenesis and cell survival under hypoxic conditions. Thus, rAAV/hTRX-PR39 may provide a novel therapeutic method for the treatment of ischemia.

\section{Acknowledgements}

The study was supported by a grant from the National Natural Science Foundation of China (no. 30970992). The authors thank Professor Qingyong Liu (Jinan Central Hospital Affiliated with Shandong University, Jinan, China) for providing support and assistance with regard to the study design and the provision of reagents; Shibao Zhang, Bo Liu and Shuangqing Liu (Jinan Central Hospital Affiliated with Shandong University) for their assistance in data collection, statistical analyses and image acquisition; Professor Jianzhong Bi (Second Affiliated Hospital of Shandong University, Jinan, China) for providing research guidance; and Professor Aiqin Song (Department of Statistics, Jining Medical College, Jining, China) for providing statistical support.

\section{References}

1. Alderazi YJ and Grotta JC: Acute antithrombotic treatment of ischemic stroke. Curr Vasc Pharmacol 12: 353-364, 2014.

2. Madonna R and Rokosh G: Insights into gene therapy for critical limb ischemia: the devil is in the details. Vascul Pharmacol 57: $10-14,2012$.

3. Navarro-Yepes J,Zavala-Flores L, Anandhan A, et al: Antioxidant gene therapy against neuronal cell death. Pharmacol Ther 142: 206-230, 2014.

4. Aoki M and Morishita R. Therapeutic angiogenesis for ischemic diseases. Nihon Rinsho 64: 762-768, 2006 (In Japanese).

5. Su H, Joho S, Huang Y, et al: Adeno-associated viral vector delivers cardiac-specific and hypoxia-inducible VEGF expression in ischemic mouse hearts. Proc Natl Acad Sci USA 101: 16280-16285, 2004.

6. Simonato M, Bennett J, Boulis NM, et al: Progress in gene therapy for neurological disorders. Nat Rev Neurol 9: 277-291, 2013.

7. Spector A, Yan GZ, Huang RR, McDermott MJ, Gascoyne PR and Pigiet V: The effect of $\mathrm{H}_{2} \mathrm{O}_{2}$ upon thioredoxin-enriched lens epithelial cells. J Biol Chem 263: 4984-4990, 1988.

8. Anbanandam A, Albarado DC, Tirziu DC, Simons M and Veeraraghavan S: Molecular basis for proline- and arginine-rich peptide inhibition of proteasome. J Mol Biol 384: 219-227, 2008

9. Borghouts C, Kunz C, Delis N and Groner B: Monomeric recombinant peptide aptamers are required for efficient intracellular uptake and target inhibition. Mol Cancer Res 6: 267-281, 2008.

10. Muinck ED, Nagy N, Tirziu D, et al: Protection against myocardial ischemia-reperfusion injury by the angiogenic Masterswitch protein PR 39 gene therapy: the roles of HIF1alpha stabilization and FGFR1 signaling. Antioxid Redox Signal 9: 437-445, 2007

11. Gao Y,Lecker S, Post MJ, et al: Inhibition of ubiquitin-proteasome pathway-mediated I kappa B alpha degradation by a naturally occurring antibacterial peptide. J Clin Invest 106: 439-448, 2000.

12. Gerber HP, Condorelli F, Park J and Ferrara N: Differential transcriptional regulation of the two vascular endothelial growth factor receptor genes. Flt-1, but not Flk-1/KDR, is up-regulated by hypoxia. J Biol Chem 272: 23659-23667, 1997.

13. Sun L, Hao Y, Nie X, Zhang X, Yang G and Wang Q: Construction of PR39 recombinant AAV under control of the HRE promoter and the effect of recombinant AAV on gene therapy of ischemic heart disease. Exp Ther Med 4: 811-814, 2012.

14. Zorko M and Langel U: Cell-penetrating peptides: mechanism and kinetics of cargo delivery. Adv Drug Deliv Rev 57: 529-545, 2005.

15. Deshayes S, Morris MC, Divita G and Heitz F: Cell-penetrating peptides: tools for intracellular delivery of therapeutics. Cell Mol Life Sci 62: 1839-1849, 2005. 
16. Ruan XY, Bi JZ, Liu QY, et al: Construction and identification of recombinant plasmids expressing hTRX-PR39. Shandong Daxue Xuebao: Yixue Ban 47: 30-34, 2009 (In Chinese).

17. Liu QY, Ruan XY, Liu XG, et al: Cloning and expression of a cDNA sequence for human thioredoxin. Xi'an Jiaotong Daxue Xuebao 15: 183-188, 2003 (In Chinese).

18. Powis G and Montfort WR: Proprties and biological activities of thioredoxins. Annu Rev Pharmacol Toxicol 41: 261-295, 2001.

19. Yegorova S, Yegorov O and Lou MF: Thioredoxin induced antioxidant gene expressions in human lens epithelial cells. Exp Eye Res 83: 783-792, 2006.

20. Kobayashi-Miura M, Nakamura H, Yodoi J and Shiota K: Thioredoxin, an anti-oxidant protein, protects mouse embryos from oxidative stress-induced developmental anomalies. Free Radic Res 36: 949-956, 2002.

21. Das SK, Sharma NK, Hasstedt SJ, et al: An integrative genomics approach identifies activation of thioredoxin/thioredoxin reductase-1-mediated oxidative stress defense pathway and inhibition of angiogenesis in obese non diabetic human subjects. J Clin Endocrinol Metab 96: E1308-E1313, 2011.

22. Umekawa T, Sugiyama T, Kihira T, et al: Overexpression of thioredoxin-1 reduces oxidative stress in the placenta of transgenic mice and promotes fetal growth via glucose metabolism. Endocrinology 149: 3980-3988, 2008.

23. Post MJ, Sato K, Murakami M, et al: Adenoviral PR39 improves blood flow and myocardial function in a pig model of chronic myocardial ischemia by enhancing collateral formation. Am J Physiol Regul Integr Comp Physiol 290: R494-R500, 2006.

24. Li J, Post M, Volk R, et al: PR39, a peptide regulator of angiogenesis. Nat Med 6: 49-55, 2000.

25. Sun L, Hao Y, Nie X, et al: Recombinant AAV-PR39-mediated hypoxia-inducible factor 1 alpha gene expression attenuates myocardial infarction. Int J Mol Med 33: 171-177, 2014.
26. Simons M: Integrative signaling in angiogenesis. Mol Cell Biochem 264: 99-102, 2004.

27. Wen YA, Yu XL, Xia QF, Cen D, Liu JB and Tu ZG Macrophage-specific overexpression of antimicrobial peptide PR-39 inhibits intracellular growth of Salmonella enterica serovar Typhimurium in macrophage cells. Int J Antimicrob Agents 34: 315-321, 2009.

28. Rodríguez-Martínez S, Cancino-Diaz JC, Vargas-Zuñiga LM and Cancino-Diaz ME: LL-37 regulates the overexpression of vascular endothelial growth factor (VEGF) and c-IAP-2 in human keratinocytes. Int J Dermatol 47: 457-462, 2008.

29. Ramanathan B, Wu H, Ross CR and Blecha F: PR-39, a porcine antimicrobial peptide, inhibits apoptosis: Involvement of caspase-3. Dev Comp Immunol 28: 163-169, 2004.

30. Shen F, Kuo R, Milon-Camus M, et al: Intravenous delivery of adeno-associated viral vector serotype 9 mediates effective gene expression in ischemic stroke lesion and brain angiogenic foci. Stroke 44: 252-254, 2013

31. Shan H, Ji D, Barnard AR, Lipinski DM, You Q, Lee EJ, Kamalden TA et al: AAV-mediated gene transfer of human $\mathrm{x}$-linked inhibitor of apoptosis protects against oxidative cell death in human RPE cells. Invest Ophthalmol Vis Sci 52: 9591-9597, 2011

32. Wang L, Blouin V, Brument N, Bello-Roufai M and Francois A: Production and purification of recombinant adeno-associated vectors. Methods Mol Biol 807: 361-404, 2011.

33. Bockstael O, Foust KD, Kaspar B and Tenenbaum L: Recombinant AAV delivery to the central nervous system. Methods Mol Biol 807: 159-177, 2011

34. Ruan XY, Yuan ZG, Du YF, Yang GX and Wang QY: Recombinant adeno-associated virus delivered human thioredoxin-PR39 prevents hypoxia-induced apoptosis of ECV304 cells. Neural Regen Res 7: 708-713, 2012. 